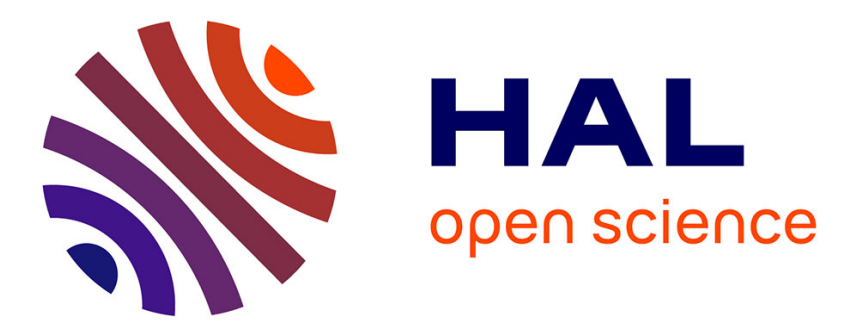

\title{
Decoupling vigour and quality in the autumn colours game: Weak individuals can signal, cheating can pay \\ Marco Archetti
}

\section{To cite this version:}

Marco Archetti. Decoupling vigour and quality in the autumn colours game: Weak individuals can signal, cheating can pay. Journal of Theoretical Biology, 2009, 256 (3), pp.479. 10.1016/j.jtbi.2008.10.012 . hal-00554518

\section{HAL Id: hal-00554518 \\ https://hal.science/hal-00554518}

Submitted on 11 Jan 2011

HAL is a multi-disciplinary open access archive for the deposit and dissemination of scientific research documents, whether they are published or not. The documents may come from teaching and research institutions in France or abroad, or from public or private research centers.
L'archive ouverte pluridisciplinaire HAL, est destinée au dépôt et à la diffusion de documents scientifiques de niveau recherche, publiés ou non, émanant des établissements d'enseignement et de recherche français ou étrangers, des laboratoires publics ou privés. 


\section{Author's Accepted Manuscript}

Decoupling vigour and quality in the autumn colours game: Weak individuals can signal, cheating can pay

Marco Archetti

PII: S0022-5193(08)00549-3

DOI: doi:10.1016/j.jtbi.2008.10.012

Reference: YJTBI5341

To appear in: $\quad$ Journal of Theoretical Biology

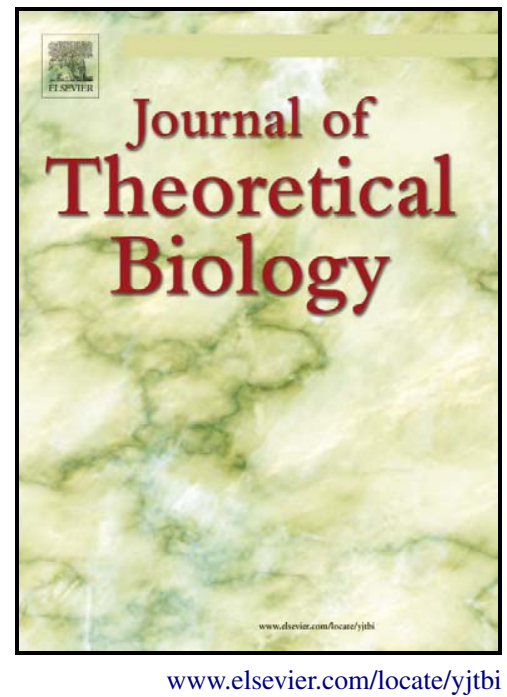

Received date: $\quad 4$ September 2008

Revised date: $\quad 19$ October 2008

Accepted date: 20 October 2008

Cite this article as: Marco Archetti, Decoupling vigour and quality in the autumn colours game: Weak individuals can signal, cheating can pay, Journal of Theoretical Biology (2008), doi:10.1016/j.jtbi.2008.10.012

This is a PDF file of an unedited manuscript that has been accepted for publication. As a service to our customers we are providing this early version of the manuscript. The manuscript will undergo copyediting, typesetting, and review of the resulting galley proof before it is published in its final citable form. Please note that during the production process errors may be discovered which could affect the content, and all legal disclaimers that apply to the journal pertain. 
Decoupling vigour and quality in the autumn colours game: weak individuals can signal, cheating can pay

MARCO ARCHETTI

Department of Zoology

Oxford University

South Parks Road, OX1 3PS

Oxford, UK

Phone: +44 (0)1865 271207

E-mail: marco.archetti@zoo.ox.ac.uk 


\begin{abstract}
According to the coevolution theory, autumn colours are a warning signal to insects, signalling the level of chemical defenses or availability of nutrients. Because in the original model tree vigour and defenses were positively correlated, it is not clear whether signalling would still be stable when they are decoupled, and the fact that weak trees often display bright autumn colours is usually presented as evidence against the coevolution theory. I show that in a theoretical model of insect-tree coevolution, signalling is still stable when vigour and defenses are decoupled. Weak trees can signal. Moreover, partial cheating is possible. The different equilibria depend on the importance of vigour and defenses against insect attack, of vigour in the production of the signal, and of pleiotropic effects between colour and defenses. These results provide precise predictions than can be used for planning future empirical test.
\end{abstract}

Keywords: Autumn colours; Signalling; Coevolution; Chemical defenses; Tree vigour; Handicap principle; Game Theory. 


\section{Introduction}

\section{Autumn colours}

The adaptive value of the autumn colours of leaves is still a matter of controversy (Ougham et al., 2008). Autumn colours are produced mainly by carotenoids (yelloworange) and anthocyanins (red-purple). While carotenoids, which are present all year, may become visible because of the seasonal breakdown of chlorophyll, anthocyanins are actively produced in autumn (Sanger, 1971; Lee 2002a; Lee 2002b; Lee \& Gould, 2002; Archetti et al., 2008). What use is the production of a pigment in leaves that are about to be shed?

Red may protect the leaf from the damaging effects of light at low temperatures (photo-inhibition and photo-oxidation), allowing a more efficient resorption of nutrients, especially nitrogen (the photoprotection theory: Pringsheim, 1879-1882;

Gould et al., 1995; Hoch et al., 2001; Feild et al., 2001; Lee et al., 2003).

Alternatively, red might be a warning signal of the status of the tree (indicating high levels of defenses or low nutritional capacity) to animals, particularly feeding insects like aphids (the coevolution theory: Archetti, 2000; Hamilton \& Brown, 2001, Archetti \& Brown, 2004). Other hypotheses are also possible (Archetti, 2008) but very few tests are available (Ougham et al., 2008; Archetti et al., 2008).

The coevolution theory

According to the coevolution theory red is a signal of the status of the tree to insects that migrate to (or move among) the trees in autumn: (i) insects migrating to the trees 
in autumn avoid red leaves and colonise preferentially green leaves; (ii) trees with red leaves have better chemical defenses or worst nutritional capacity or any other characteristic that induces a lower fitness in the insects. In this scenario, therefore, colour and preference coevolve in an arms race: autumn colours are an adaptation of the trees to reduce their parasite load and insect preference for green is an adaptation to find the most suitable host trees.

There is no debate on the importance of the first point (colour preference), although it is debated whether insects actually avoid red leaves (the tests performed so far seem to support this claim: Archetti \& Leather, 2005; Hagen et al., 2003; Hagen et al., 2004; Karageorgou \& Manetas, 2006; Doering et al., 2008; see also Furuta, 1986; contrasting evidence has been reported by Schaefer \& Rolshausen, 2007; Rolshausen \& Schaefer, 2007; reviewed by Archetti et al., 2008). This is not the point of this paper.

The second main point of the theory however, the link between autumn colour and defenses, is much more controversial. According to the coevolution theory, autumn colours are a signal that the tree has strong chemical defences or lower nutritional quality, or any other characteristic that would induce a lower fitness in the insects. This is often confused with the following statement: that the trees with red leaves are the most vigorous ones. This misunderstanding has created much confusion, and because there is some evidence that weak trees often display strong autumn colours (Schaberg et al., 2003; Ougham et al., 2005), it may seem to undermine the coevolution theory. 


\section{Vigour and quality}

This misunderstanding (that trees with red leaves must be the most vigorous ones) is probably due to the fact that in the original model of the coevolution theory (Archetti, 2000) I did not decouple vigour from defenses. In that model I assumed that the trees with high levels of defenses were always the most vigorous ones. This creates a system in which the trees that signal are the ones with high levels of defenses - and these are also the most vigorous ones (an example of handicap signalling: Zahavi, 1975; Grafen, 1990).

That model (Archetti 2000) assumed that the vigour of the tree was positively correlated with the level of defenses. This is not necessary, however. As Archetti \& Brown (2004) pointed out, stronger autumn colours need not be associated with more vigorous trees. Hamilton \& Brown (2001) were also careful on this point, and even suggested that it might be the weaker trees that need to signal more. The link must be with the quality of the tree that is relevant for the insects, that is chemical defenses or nutritional capacity or any characteristic that may affect insect fitness. And this is not necessarily positively correlated with tree vigour.

Even Ougham et al. (2005) in their criticism of the coevolution theory suggested that the theory can still work if the weak trees are the ones more in need to avoid parasites. Indeed this fact is not controversial in the theory of biological signalling: the requirement for the evolutionary stability of signalling is that the ratio of the fitness cost of the signal and the benefit received be lower in individuals giving stronger signal, either because the signal is less costly for a signaller of high quality or because individuals give stronger signals when in greater need (Maynard-Smith \& Harper, 2003). 
In the classical model of biological signalling, developed in the context of animal sexual selection, the quality that is relevant for the signaller (the male) and the quality that is relevant for the receiver (the female) are the same: the male's vigour, which affects both the cost of the signal for the male and the fitness of the female. In the autumn colours signalling game, however, the quality that is relevant for the cost of the signal (tree vigour) is not the same that is relevant for the receiver (the level of chemical defenses); a vigorous tree is not necessarily one with high defenses (or low nutrition or anything that affects negatively the fitness of the insects), and a weak tree is not necessarily one with low defenses.

\section{Rationale of the paper}

My purpose is to analyse the autumn colours signalling game decoupling the quality that affects the cost of the signal (the vigour of the tree) and the quality that is relevant for the receiver (the level of defenses or any quality that affects the fitness of the insects). In the original model of the coevolution theory (Archetti, 2000) these two qualities were coupled (vigorous trees were the ones with higher levels of defenses). What happens when vigour and defenses are decoupled? Is signalling still stable in this case? Is it still honest? (that is, do the receivers benefit from trusting the signal?). Are the trees that display more autumn colours also more vigorous?

\section{The model}

I model the following scenario: trees are colonised by insects looking for a host in autumn. The fitness of a tree is affected by the damage induced by the insects and by 
the cost of autumn colours; the fitness of the insects depends on the quality of the tree they colonise. Trees can signal their status to the insects using leaf colour. Insects must find the most suitable hosts but cannot perceive the vigour nor the defenses of the trees - they can only perceive the colour. The problem is to understand whether a signalling system in which the trees use red as a warning signal, and insects trust the signal, is stable.

Strategies

I use a discrete model in which a tree is assigned two possible values (high and low) for three parameters: vigour, defenses and colour.

Colour (s): a tree can be either red (S) or green (s) in autumn.

$\operatorname{Vigour}(v)$ : a tree can be either strong $(\mathbf{V})$ or weak $(\mathbf{v})$. This is the quality from the point of view of the tree itself: it can affect the tree's cost for the production of the signal and its fitness in response to insect attack but does not affect insect fitness at all.

Defenses $(d)$ : a tree can have high (D) or low (d) defenses against insects. I will talk about defenses for simplicity, but this does not necessarily mean chemical defenses: it could be any characteristic of the tree that affects insect fitness, for example low nutritional capacity (less nutrients available to insects) would be equivalent to high chemical defenses.

Vigour and defenses are decided by nature (the environment or genetically determined). I assume an equal frequency of $\mathbf{d}$ and $\mathbf{D}$ trees $\left(f_{\mathbf{d}}=f_{\mathbf{D}}=1 / 2\right)$ and of $\mathbf{v}$ and $\mathbf{V}$ trees $\left(f_{\mathbf{v}}=f_{\mathbf{v}}=1 / 2\right)$. Because what matters is their relative value, this assumption does not affect the generality of the results and only means that low defences are as likely as 
high defences, and weak trees are as likely as strong trees; that is the threshold defining high and low is exactly the average value of the population.

In the original model of the coevolution theory (Archetti, 2000) vigour and defenses were considered to be the same parameter (named "quality"): as a result there were only four possible tree strategies. In this model vigour and defenses can be decoupled, that is a tree can have, for example, high vigour and low defenses. Given vigour and defenses (chosen by nature), a tree's strategy consists in being $\mathbf{s}$ or $\mathbf{S}$ according to its own vigour $(v)$ and defenses $(d)$. I assume that the probability $x_{i v d s}$ of being $\mathbf{S}$ for a $v d$ tree playing strategy $i$ is either 0 or 1 . As a consequence there are sixteen possible tree strategies $i$ (Table 1 ). For example strategy $i=2$ means signalling (red) $\mathbf{S}$ only if vigorous and with high levels of defenses (VD).

Insects cannot perceive directly vigour nor defenses but must rely on the signal (colour) to choose their host. I assume there are only three possible insect strategies $j$ : preference for $\mathbf{s}$, preference for $\mathbf{S}$ and no preference (Table 2). With this model of preference, there are two indeterminate cases when there is only one kind of tree colour and insects with a preference for the other colour: $(i=1, j=3$ and $i=16, j=2)$. I assume that insects in these two cases fail to find a host and their fitness is zero.

\section{Fitness}

The rate of survival of a $v d s$ tree in autumn is $\lambda_{v d s}$. In this phase fitness depends only on the cost of the signal. The rate of survival of a $v d s$ tree after autumn is $\phi_{v d s}$. In this phase fitness depends on the cost of being colonised by insects. Therefore the fitness of a tree playing strategy $i$ can be defined by 


$$
T_{i}=\sum_{v d s} f_{v} f_{d} x_{i v d s} \lambda_{v d s} \phi_{v d s}
$$

Where

$$
\left.\phi_{v d s}=\sum_{j} a_{j}\{\underbrace{\left(1-y_{j s}\right)}_{\begin{array}{c}
\text { colour } \\
\text { avoided }
\end{array}}+\underbrace{y_{j s}[\overbrace{\frac{N f_{s}-1}{N f_{s}}+\overbrace{\frac{1}{N f_{s}}\left(1-\sigma_{v d}^{\prime}\right)}^{\text {individual }}}^{\text {avoided }} \begin{array}{c}
\text { individual } \\
\text { chosen }
\end{array}}_{\begin{array}{c}
\text { colour } \\
\text { chosen }
\end{array}}\}\right\}
$$

in which $a_{j}$ is the frequency of insect strategy $j, \sigma_{v d}^{\prime}$ is the cost $\left(0<\sigma_{v d}^{\prime}<1\right)$ due to the attack of the insect population on $v d$ trees and $N$ is the number of trees on which the attack is concentrated. For each kind of insect $j$, with frequency $\left(1-y_{j s}\right)$ colour $s$ is avoided and the fitness of the tree is 1 ; with frequency $y_{j s}$ the insects accept colour $s$ : in this case a tree of that colour is chosen with probability $1 / N f_{s}$ and in this case the tree has fitness $\left(1-\sigma_{v d}^{\prime}\right)$; with probability $\left(N f_{s^{-}}-1\right) / N f_{s}$ the tree is not chosen and has fitness 1 .

$N$ can be incorporated in $\sigma_{v d}^{\prime}$ without loss of generality and we can substitute $\sigma_{v d}^{\prime}$ with $\sigma_{v d}$, which is the cost due to part of the insect population that attacks the tree. $\phi_{v d s}$ reduces to $\phi_{v d s}=\sum_{j} a_{j}\left\{1-y_{j s} \sigma_{v d} / f_{s}\right\}$ 
The fitness of an insect playing strategy $j$ is defined by

$$
A_{j}=\sum_{s} y_{j s} \sum_{d} f_{d s} \gamma_{d} / f_{s}-\varepsilon_{j}
$$

where $f_{d s}$ is the frequency of $d s$ trees in the population and $f_{s}$ is the frequency of $s$ trees in the population; $\gamma_{d}$ is the fitness of an insect on a $d$ tree. I assume that insect fitness does not depend on the tree's vigour $v$ or colour $s$ but only on its defenses $d$. $\varepsilon_{j}$ is a small cost paid by choosy insects $\left(\varepsilon_{j}>0\right.$ for $j=2,3 ; \varepsilon_{j}=0$ for $\left.j=1\right)$.

The following assumptions can be considered true in any situation: insects perform better on trees with low defences $\left(\gamma_{\mathbf{D}}<\gamma_{\mathbf{d}}\right)$; the damage due to insects is lower in trees with high levels of defences $\left(0<\sigma_{v \mathbf{D}}<\sigma_{v \mathbf{d}}\right)$ and in vigorous trees $\left(0<\sigma_{\mathbf{v} d}<\sigma_{\mathbf{v} d}\right)$. Other conditions will vary and decide the stability of the strategies.

\section{Stability}

Trees can prefer to pay the cost of having insects rather than the cost of producing the signal either because producing a signal is too costly (for example because of the loss of photosynthesis or the cost of producing anthocyanins) or because the cost of insects is low (for example if the defenses are so effective that no damage is done by the insects). Or the trees can prefer to avoid the cost of having insects and pay the cost of producing the signal, either because producing the signal has a low cost or because the cost of being invaded by insects is high (for example if the defenses are not effective). Different strategies, therefore, might be stable under different parameters.

The aim of the analysis is to find couples of strategies, one for the trees $\left(i^{*}\right)$ and one for the insects $\left(j^{*}\right)$, that are evolutionarily stable (ESS - Maynard-Smith 
1982), that is couples $\left(i^{*}, j^{*}\right)$ that satisfy the conditions $T_{\mathrm{i}^{*}}>T_{\mathrm{i}}$ and $A_{\mathrm{j}^{*}}>A_{\mathrm{j}}$ for all other insect strategies $j$ and all other tree strategies $i$. Therefore the fitness of all tree strategies is calculated with each insect strategy $j^{*}$ fixed in the population $\left(f_{j *}=1\right)$ to check whether, and for which parameters, a tree strategy $i^{*}$ satisfies the condition $T_{\mathrm{i}^{*}}>T_{\mathrm{i}}$ for all other tree strategies $i$. At the same time, the fitness of all the insect strategies is calculated with each tree strategy $i^{*}$ fixed in the population $\left(f_{i *}=1\right)$ to check whether, and for which parameters, an insect strategy $j^{*}$ satisfies the condition $A_{\mathrm{j}^{*}}>A_{\mathrm{j}}$ for all other insect strategies $j$. The stability analysis was performed with Mathematica 6.0 (Wolfram Research).

\section{Results}

Vigour linked to defenses

Consider, first, the following case: constrain $\mathbf{v}$ to $\mathbf{d}$ and $\mathbf{V}$ to $\mathbf{D}$, and call them $\mathbf{q}(=\mathbf{v}$ and $\mathbf{d})$ and $\mathbf{Q}(=\mathbf{V}$ and $\mathbf{D})$. With this constraint the model is identical to the original coevolution model (Archetti 2000); vigour and defenses are not decoupled. This means that only the following tree strategies are allowed: Never signal; Signal if $\mathbf{Q}$; Signal if q; Always signal.

There is a non signalling-equilibrium ( $j=1$; Trees: Never signal) and a signalling equilibrium $(j=2$; Trees: signal if $\mathbf{Q})$. The signalling equilibrium is stable if

$$
\begin{aligned}
& \sigma_{\mathrm{q}} / 2<1-\lambda_{\mathrm{qS}} / \lambda_{\mathrm{qs}} \\
& \sigma_{\mathrm{Q}} / 2>1-\lambda_{\mathrm{QS}} / \lambda_{\mathrm{Qs}}
\end{aligned}
$$


This means that the relative cost paid for the production of the signal $1-\lambda_{q \mathrm{~S}} / \lambda_{q \mathrm{~s}}$ must be smaller than the (half) cost due to insect attack for $\mathbf{Q}$ trees but the relative cost paid for the production of the signal must be higher than the (half) cost due to insect attack for $\mathbf{q}$ trees. This is a form of handicap principle (Archetti, 2000; Grafen, 1990; Zahavi, 1975). The signal is honest.

It is important to notice that at the signalling equilibrium only $\mathbf{Q}$ trees signal, therefore the actual cost for the production of the colour can even be zero (see also Maynard-Smith \& Harper, 2003). It is true that a cost prevents $\mathbf{q}$ trees from signalling, but this cost prevents them from signalling indeed, and it is not paid at equilibrium. The relevant cost is the one paid by signalling trees, and this cost can also be zero. In other words, it must be $\lambda_{\mathrm{q} s}<\lambda_{\mathrm{qs}}$, but it could well be $\lambda_{\mathrm{Q}}=\lambda_{\mathrm{Qs}}$.

This result, so far, is not different from the original model (Archetti, 2000) where $v$ and $d$ were not decoupled. The results are more interesting when we consider the complete version of the model, in which $d$ and $v$ are allowed to be decoupled.

Decoupling vigour and defenses

The stability analysis shows that signalling can be stable with insect strategy $j=2$ and five tree strategies $i: 2,5,6,8$ and 14 (Table 3). Other signalling strategies are not stable. For example with $i=4$ (signal if $\mathbf{V}$ ) only insects with no preference are stable, but with this insect strategy only tree strategy $i=1$ is stable. A non-signalling equilibrium always exists with insect strategy $j=1$ (no preference) and tree strategy $i=1$ (never signal), as in Archetti (2000). The unstable equilibria and the non- 
signalling equilibrium are trivial and will not be discussed further; in the rest of the paper I will focus on the different possible signalling equilibria.

The conditions for the stability of the insect strategy $j=2$ with the five stable tree strategies (Table 3) can be considered always true for $\sum_{j}$ small. Stability for the trees depends on the relative value of the cost for the production of the colour and on the damage due to insect attack, as in the original model (Archetti, 2000) but in a more complex way. For each tree strategy there are four conditions with the form $\sigma_{v d \mathbf{s}} / f_{\mathbf{s}}<$ or $>1-\lambda_{v d \mathbf{S}} / \lambda_{v d \mathbf{s}}$ (Table 3$)$. For $i=6$, for example, these conditions are:

$\sigma_{\text {VDs }} / 2>1-\lambda_{\text {VDS }} / \lambda_{\text {VDs }}$

$\sigma_{\mathbf{V d s}} / 2<1-\lambda_{\mathbf{V d S}} / \lambda_{\mathbf{V d s}}$

$\sigma_{\mathrm{vDs}} / 2>1-\lambda_{\mathrm{vDS}} / \lambda_{\mathrm{vDs}}$

$\sigma_{\mathbf{v d s}} / 2<1-\lambda_{\mathbf{v d S}} / \lambda_{\mathbf{v d s}}$

The quantity $1-\lambda_{v d s} / \lambda_{v d \mathbf{S}}$ is the relative cost for the production of colour for a tree $\mathbf{S}$ compared to a tree $\mathbf{s}$ with the same $v d$ values. Therefore the inequality signs in Table 3 mean that the (half) cost due to insect attack must be larger $(>)$ or smaller $(<)$ than the relative cost of the production of colour for trees with the given $v d$ values. The relative cost of the defenses, instead, is not relevant for the equilibria.

Trees adopting strategy $i=2$ signal only when they are VD, that is when they are vigorous and have truly high levels of defenses. This is partial conceal because vD trees do not signal. This case is stable if (see Table 3) the cost of the signal is lower than the cost due to insect attack in VD trees but higher in all other cases.

Trees adopting strategy $i=5$ signal only when they are $\mathbf{v D}$, that is when they are weak (and have truly high levels of defenses). This is also partial conceal, because 
VD trees do not signal. This case is stable if (see Table 3) the cost of the signal is lower than the cost due to insect attack in vD trees but higher in all other cases.

From the point of view of the insects $i=2$ and $i=5$ are stable because (with $f_{\mathrm{d}}=f_{\mathrm{v}}=1 / 2$ ) insects preferring green end up on $\mathbf{D}$ trees only $1 / 3$ of the times ( $\mathbf{v D}$ in the case of $i=2$; VD in the case of $i=5$ ) while insects preferring red would always end up on $\mathbf{D}$ trees (VD in the case of $i=2 ; \mathbf{v D}$ in the case of $i=5)$ and insects with no preference would end up on $\mathbf{D}$ trees $1 / 2$ of the times. This is not the best possible equilibrium for insects (the best would be always avoiding $\mathbf{D}$ trees) but it is stable.

Trees adopting strategy $i=6$ signal if and only if they really have a high level of defenses (D); vigour is irrelevant. Therefore the insects always choose the trees with the low defenses. This case is stable if (see Table 3) the cost of the signal is lower than the cost due to insect attack in $\mathbf{D}$ trees but higher in $\mathbf{d}$ trees, irrespective of vigour. This is the optimal scenario for aphids because they always manage to avoid trees with high levels of defenses.

Trees adopting strategy $i=8$ signal if and only if they are not vd (therefore VD, Vd and vD signal). This is partial deceit (dishonest) because Vd trees signal. This case is stable if (see Table 3) the cost of the signal is lower than the cost due to insect attack in all trees except vd trees (in which this cost is higher).

Trees adopting strategy $i=14$ signal if and only if they are not Vd (therefore VD, vd and vD signal). This is also partial deceit because vd trees signal. This case is stable if (see Table 3) the cost of the signal is lower than the cost due to insect attack in all trees except Vd trees (in which this cost is higher).

From the point of view of the insects $i=8$ and $i=14$ are stable because insects preferring green never end up on $\mathbf{D}$ trees, although $1 / 3$ of the times (with $f_{\mathrm{d}}=f_{\mathrm{q}}=1 / 2$ ) they avoid $\mathbf{d}$ trees too ( $\mathbf{V d}$ in the case of $i=8$; $\mathbf{v d}$ in the case of $i=14)$; insects 
preferring red would end up on $\mathbf{D}$ trees $2 / 3$ of the times) and insects with no preference would end up on $\mathbf{D}$ trees $1 / 2$ of the times.

Therefore, in summary, when vigour and defenses are decoupled, signalling can still be stable but it is not necessarily honest. Trees with low defenses (d) can signal. Moreover, signalling is not restricted to vigorous trees. Weak trees (v) can signal. These equilibria are possible under different conditions as discussed in the next section.

\section{Discussion}

\section{Different equilibria with different effects}

I will discuss four possible effects (see Table 4) that may affect the likelihood of these combinations. There may be more, but these seem to be the ones relevant for the model, given the biological scenario involyed.

\section{Pleiotropy between colour and defenses.}

If the cost of the production of the colour is lower for $\mathbf{D}$ trees than for $\mathbf{d}$ trees, or if $\mathbf{D}$ trees are necessarily more coloured than $\mathbf{d}$ trees, for example because of a pleiotropic effect between colour and defenses (red pigments and chemical defenses are produced by the same biochemical pathway), then, everything else being equal (no effect of defenses on insect attack, no effect of vigour on insect attack, no effect of vigour on the cost of the signal), D trees are expected to signal more than $\mathbf{d}$ trees. Pleiotropy, therefore, make all strategies more likely to be stable, but especially strategy $i=6$ (see Table 4) 


\section{Defenses affect the fitness of the tree after insect attack.}

If $\mathbf{d}$ trees are more susceptible to the damages induced by insects than $\mathbf{D}$ trees, then, everything else being equal (no pleiotropic effects, no effect of vigour on insect attack, no effect of vigour on the cost of the signal), they should signal more than D trees because they need more to avoid insects. This effect may be more important in weak trees (v) if, in addition, weak trees are more susceptible to the effects of insect attack. If high levels of chemical defenses are efficient in protecting the tree after insect attack, therefore, the likelihood of all signalling strategies is reduced, but especially the likelihood of the honest strategy $i=6$ (see Table 4 ).

\section{Vigour affects the cost of the signal.}

If $\mathbf{V}$ trees pay a lower cost for the signal than $\mathbf{v}$ trees, then, everything else being equal (no pleiotropic effects, no effect of vigour on insect attack, no effect of defenses on insect attack), $\mathbf{V}$ trees are expected to signal more than $\mathbf{v}$ trees. If the vigour of the tree affects significantly the cost of the signal, therefore, strategies $i=2$ and $i=8$ are more likely and strategies $i=5$ and $i=14$ less likely (see Table 4 ).

\section{Vigour affects the fitness of the tree after insect attack.}

If $\mathbf{v}$ trees are more susceptible to the damages induced by insects than $\mathbf{V}$ trees, then, everything else being equal (no pleiotropic effects, no effect of defenses on insect attack, no effect of vigour on the cost of the signal), they should signal more because they need to avoid insects more than $\mathbf{V}$ trees. This effect may be more important in trees with low defenses (d) if, in addition, $\mathbf{d}$ trees are more susceptible to the effects of insect attack. If the vigour of the tree affects significantly the effect of insect attack 
on the tree's fitness, therefore, strategies $i=2$ and $i=8$ are less likely and strategies $i=5$ and $i=14$ more likely (see Table 4 ).

These four effects (Table 4) are likely to occur at the same time, therefore they must be weighted according to their relative importance. Different combinations of the four effects may lead to different signalling equilibria, or in some cases to no signalling at all.

Evidence for the different effects and possible tests

A pleiotropic effect that leads to an association between colour and defenses is not unlikely (Ougham et al., 2005) but showing that trees with more (or more intense) red leaves have more chemical defenses would not reveal a pleiotropic effect. Moreover it must be kept in mind that chemical defenses are not the only possibility. High levels of defenses would be equivalent, in the model, to poor nutritional capacity for the insects. This can perhaps be measured directly, or as an alternative approach, the growth rates of insects could be an indirect measure of $d$ (this approach has been used by Ramirez et al., 2008). There is little doubt that the quality of the tree as a host is crucial for the insects: Moran \& Witham (1990), for example, report a differential survival of aphids on Populus ranging between $0 \%$ and $78 \%$. The importance of vigour and defenses against insects for the fitness of the tree, however, is more difficult to measure. It is possible, and there is evidence for this (Ougham et al., 2005), that tree vigour affects the impact of insect attack on the tree and that weak trees need more than healthy trees to avoid parasites. On the other hand it is likely that vigorous trees can afford the production of the signal more easily than weak trees. 
What matters is the relative importance of the four effects combined. This will vary from species to species, and possibly even within each species. Measuring these effects is difficult, but it might be possible to estimate their relative importance.

\section{Honesty and cheating}

Seven combinations of parameters exist for the five stable strategies, for which, given a certain $v$, signalling occurs only with one value of $d$ or, given a certain $d$, signalling occurs only with one value of $v$.

The following cases are honest (the true level of defenses is signalled):

D signals and $\mathbf{d}$ does not, irrespective of vigour $(i=6)$.

VD signals and Vd does not ( $i=2$ and 14).

vD signals and vd does not $(i=5$ and 8$)$.

The following cases are dishonest ( $\mathbf{d}$ trees can sometimes signal):

Vd signals and vd does not $(i=8)$.

vd signals and Vd does not $(i=14)$.

The following cases are concealing ( $\mathbf{D}$ trees do not always signal):

VD signals and $\mathbf{v D}$ does not $(i=2)$.

vD signals and VD does not $(i=5)$.

All the five strategies except $i=6$ are a mixture of these cases, and therefore only partially honest. Strategies $i=8$ and $i=14$ are partially dishonest and strategies $i=2$ and $i=5$ are partially concealing.

Weak trees can signal 
There is some evidence that the extent and earliness of onset of red coloration is positively correlated with foliar nitrogen deficiency in some cases (Schaberg et al., 2003; Ougham et al., 2005): this suggests that in these cases it is the weak trees that signal. As the model shows, this does not contradict the coevolution theory: the theory is still valid if it is the weakest trees that have a higher need of avoiding parasites. Weak trees can signal. In other cases (Hagen et al., 2004) there is evidence that it is the vigorous trees that display more intense colours. These apparently opposite results can be explained in the light of the present model: they might be the different stable outcomes of signalling under different conditions. As I have shown the relative importance of defenses (on insect fitness and on tree fitness), vigour (for the tree against insect attack) and pleiotropy (between colour and defenses) will affect the stable signalling equilibria. If we want to develop rigorous tests of the theory we must be able to weight the relative importance of these four (and possibly other) effects as suggested by the model.

It should also be noted that support for a positive correlation between vigour and autumn colours (Hagen et al., 2004) was found in a species with yellow, rather than red, autumn colours. Yellow is due to the breakdown of chlorophyll rather than to a de novo production of pigments in autumn (Archetti et al. 2008), and of red and yellow autumn colours are likely to be different. In the case of Betula pubescens in Northern Norway it is possible that the loss of photosynthesis due to an earlier breakdown of chlorophyll represents an important cost that only vigorous trees can afford, because the growing season is very short, but in other species the cost of reduced photosynthesis might be irrelevant (Ougham et al. 2005). 


\section{Relevance for comparative analyses}

The model has also implications for comparative analysis. The weighted combinations of the four effect can lead, in some cases, to no signalling at all (the non signalling equilibrium is always a possible alternative) even in the presence of insects. It is questionable, therefore, how powerful interspecific comparative analyses can be in revealing a correlation between insect presence and presence of autumn colour (Hamilton \& Brown, 2001) if the details of the system (importance of vigour and defenses on tree fitness, importance of pleiotropy between colour and defenses, importance of vigour for the production of the colour) are unknown. It might be that a coevolutionary arms race is going on between insects and trees with parameters (vigour, quality, pleiotropy) that do not lead to any stable signalling equilibrium. We should not necessarily expect, therefore, that autumn colours are present in all cases where a coevolutionary interaction occurs.

\section{Conclusion}

It is clear that signalling is possible when vigour and defenses are decoupled.

Signalling is not necessarily completely honest: trees with low defenses can sometimes signal. And it is not necessarily restricted to vigorous trees: weak trees can sometimes signal. The different possible equilibria are stable under different conditions, depending on the relative importance of vigour and defenses against insect attack, of vigour in the production of the signal, and of pleiotropic effects between colour and defenses. These results could help in planning future experimental tests on the coevolution theory. The model might also be applied to other cases of signalling 
in which the quality of the signaller is decoupled from the quality sought by the receiver.

\section{Acknowledgements}

I am supported by a Junior Research Fellowship from St. John's College, Oxford, and a Long-Term Fellowship from the Human Frontier Science Program. 


\section{References}

Archetti, M. 2000. The origin of autumn colours by coevolution. J. Theor. Biol. 205, 625630.

Archetti, M. 2008. Classification of hypotheses on the evolution of autumn colours. Oikos, in press.

Archetti, M., Brown, S.P. 2004. The coevolution theory of autumn colours. Proc. Royal Soc. B 271, 1219-1223.

Archetti, M., Döring, T.F., Hagen, S.B., Hughes, N.M., Leather, S.R. et al. 2008.

Unravelling the Evolution of Autumn Colours: An Interdisciplinary Approach. Trends in Ecology and Evolution, in press.

Archetti, M., Leather, S.R. 2005. A test of the coevolution theory of autumn colours: colour preference of Rhopalosiphum padi on Prunus padus. Oikos 110, 339-343.

Feild, T.S., Lee, D.W., Holbrook, N.M. 2001. Why leaves turn red in autumn. The role of anthocyanons in senescing leaves of red-osier dogwood. Plant Physiol. 127, 566-574.

Furuta, K. 1986. Host preference and population dynamics in an autumnal population of the maple aphid, Periphyllus californiensis Shinji (Homoptera, Aphididae). J. Appl. Entom. 102, 93-100.

Gould, K.S., Kuhn, D.N., Lee, D.W., Oberbauer, S.F. 1995. Why leaves are sometimes red. Nature 378, 241-242.

Grafen, A. 1990. Biological signals as handicaps. J. Theor. Biol. 144, 517-546.

Hagen, S.B., Folstad, I., Jakobsen, S.W. 2003. Autumn colouration and herbivore resistance in mountain birch (Betula pubescens). Ecol. Lett. 6, 807-811.

Hagen, S.B., Debeausse, S., Yoccoz, N.G., Folstad, I. 2004. Autumn colouration as a signal of tree condition. Proc. Royal Soc. B 271, Suppl 4, S184-185 
Hamilton, W.D., Brown, S.P. 2001. Autumn tree colours as a handicap signal. Proc. Royal Soc. B 268, 1489-1493.

Hoch, W.A., Zeldin, E.L., McCown, B.H. 2001. Physiological significance of anthocyanins during autumnal leaf senescence. Tree physiol. 21, 1-8.

Karageorgou, P., Manetas Y. 2006. The importance of being red when young, anthocyanins and the protection of young leaves of Quercus coccifera from insect herbivory and excess light. Tree Physiol. 26, 613-621.

Lee, W.D. 2002a. Anthocyanins in leaves, distribution, phylogeny and development. Adv. Bot. Res. 37, 37-53.

Lee, D.W. 2002b. Anthocyanins in autumn leaf senescence. Adv. Bot. Res. 37, 147-165.

Lee, D.W., Gould, K.S. 2002. Anthocyanins in leaves and other vegetative organs: An introduction. Adv. Bot. Res. 37, 1-16.

Maynard-Smith, J. 1982. Evolution and the Theory of Games. Cambridge University Press, Cambridge, U.K.

Maynard-Smith J., Harper, D. 2003 Animal Signals. Oxford University Press, Oxford.

Moran, N.A., Witham, T.G. 1990. Different colonisation of resistant and susceptib le host plants: Pemphigus and Populus. Ecology 71, 1059-1067.

Ougham, H.J., Morris, P., Thomas, H. 2005. The colors of autumn leaves as symptoms of cellular recycling and defenses against environmental stresses. Curr. Topics Dev. Biol. $66,135-160$.

Ougham, H.J., Thomas, H., Archetti, M. 2008. Origin and evolution of autumn colours. New Phytol., 175, 9-13.

Pringsheim, N. 1879-1882. Ueber Lichtwirkung und Chlorophyllfunction in der Planze. Jahrbuch fuer Wissenschaftliche Botanik, Volumes 12/13, G.Boratrager, Berlin. 
Ramirez, C., Lavandero, B., Archetti, M. 2008. Coevolution and the adaptive value of autumn tree colours: colour preference and growth rates of a southern beech aphid. J. Evol. Biol. 21, 49-56.

Rolshausen, G., Schaefer, H.M. 2007. Do aphids paint the tree red? Can herbivore resistance or photoprotection explain colourful leaves in autumn? Plant Ecol. 191, 77-84.

Sanger, J. 1971. Quantitative investigations of leaf pigments from their inception in buds through autumn coloration to decomposition in falling leaves. Ecology 52, 1075-1089.

Schaberg, P.G., Van den Berg, A.K., Murakami, P.F., Shane, J.B., Donnelly, J.R. 2003. Factors influencing red expression in autumn foliage of sugar maple trees. Tree Physiol. $23,325-333$.

Schaefer, H.M., Rolshausen, G. 2007. Aphids do not attend to leaf colour as handicap signal, but to the handicap of reproductive investment. Biol. Lett. 3, 1-4.

Zahavi, A. 1975. Mate selection - A selection for a handicap. J. Theor. Biol. 53, 205-214. 


\section{Table 1}

The probability $\left(\mathrm{x}_{i v d \mathbf{S}}\right)$ of being $\mathbf{S}$ for a tree $i$ according to its conditions $v$ and $d$. The probability of being $\mathbf{s}$ is $1-\mathrm{x}_{i v d \mathbf{S}}$

\begin{tabular}{|c|c|c|c|c|c|}
\hline$i$ & $\mathbf{S}$ (red) & $\mathbf{x}_{i v \mathrm{dS}}$ & $\mathbf{x}_{\text {ivDS }}$ & $\mathbf{x}_{i \mathrm{VdS}}$ & $\mathbf{x}_{i \mathrm{VDS}}$ \\
\hline 1 & Never & 0 & 0 & 0 & 0 \\
\hline 2 & If VD & 0 & 0 & 0 & 1 \\
\hline 3 & If $\mathbf{V d}$ & 0 & 0 & 1 & 0 \\
\hline 4 & If $\mathbf{V}$ & 0 & 0 & 1 & 1 \\
\hline 5 & If $\mathbf{v D}$ & 0 & 1 & 0 & 0 \\
\hline 6 & If $\mathbf{D}$ & 0 & 1 & 0 & 1 \\
\hline 7 & If $\mathbf{v D}$ or $\mathbf{V d}$ & 0 & 1 & & 0 \\
\hline 8 & If not vd & 0 & 1 & 1 & 1 \\
\hline 9 & If vd & 1 & 0 & 0 & 0 \\
\hline 10 & If $\mathbf{v d}$ or VD & & 0 & 0 & 1 \\
\hline 11 & If $\mathbf{d}$ & & 0 & 1 & 0 \\
\hline 12 & If not $\mathbf{v D}$ & 1 & 0 & 1 & 1 \\
\hline 13 & If $\mathbf{v}$ & 1 & 1 & 0 & 0 \\
\hline 14 & If not Vd & 1 & 1 & 0 & 1 \\
\hline 15 & If not VD & 1 & 1 & 1 & 0 \\
\hline 16 & Always & 1 & 1 & 1 & 1 \\
\hline
\end{tabular}




\section{Table 2}

The probability $\left(\mathrm{y}_{j s}\right)$ of choosing $s$ for a $j$ insect

\begin{tabular}{llcc}
\hline$j$ & preference & $\mathrm{y}_{j \mathbf{s}}$ & $\mathrm{y}_{j \mathbf{S}}$ \\
\hline 1 & No preference & $f_{\mathbf{s}}$ & $f_{\mathbf{S}}$ \\
2 & Prefer green & 1 & 0 \\
3 & Prefer red & 0 & 1 \\
\hline
\end{tabular}




\section{Table 3}

The sign $\left(<\right.$ or $>$ ) of the four conditions $\sigma_{v d \mathbf{s}} / f_{\mathbf{s}}(<$ or $>) 1-\lambda_{v d \mathbf{S}} / \lambda_{v d \mathbf{s}}$ for the stability of the five tree strategies $i$ that can be stable with insect strategy $j=2$ (preference for green), and the value of $f_{\mathrm{s}}$. The conditions for the stability of insect strategy $j=2$ are also shown.

\begin{tabular}{llllllll}
\hline$i$ & S (red) & \multicolumn{3}{c}{$v d$} & $f_{\mathrm{s}}$ & insects \\
\cline { 3 - 5 } & & VD & $\mathbf{V d}$ & $\mathbf{v D}$ & $\mathbf{v d}$ & \\
\hline 2 & if VD & $>$ & $<$ & $<$ & $<$ & $3 / 4$ & $\varepsilon_{2}<\left(\gamma_{\mathbf{d}}-\gamma_{\mathbf{D}}\right) / 6$ \\
5 & if vD & $<$ & $<$ & $>$ & $<$ & $3 / 4$ & $\varepsilon_{2}<\left(\gamma_{\mathbf{d}}-\gamma_{\mathbf{D}}\right) / 6$ \\
6 & if $\mathbf{D}$ & $>$ & $<$ & $>$ & $<$ & $1 / 2$ & $\varepsilon_{2}<\left(\gamma_{\mathbf{d}}-\gamma_{\mathbf{D}}\right) / 2$ \\
8 & if not vd & $>$ & $>$ & $>$ & $<$ & $1 / 4$ & $\varepsilon_{2}<\left(\gamma_{\mathbf{d}}-\gamma_{\mathbf{D}}\right) / 2$ \\
14 & if not Vd & $>$ & $<$ & $>$ & $>$ & $1 / 4$ & $\varepsilon_{2}<\left(\gamma_{\mathbf{d}}-\gamma_{\mathbf{D}}\right) / 2$ \\
\hline
\end{tabular}




\section{Table 4}

Honesty and likelihood of the five stable signalling equilibria $i$ for trees with insect strategy $j=2$ (preference for green).

\begin{tabular}{|c|c|c|c|c|c|c|}
\hline \multirow[t]{2}{*}{$i$} & \multirow[t]{2}{*}{$\mathbf{S}$ (red) } & \multirow[t]{2}{*}{ honesty } & \multicolumn{4}{|c|}{ effects $^{a}$} \\
\hline & & & 1 & 2 & 3 & 4 \\
\hline 2 & if VD & partially concealing & + & - & + & - \\
\hline 5 & if $\mathbf{v D}$ & partially concealing & + & - & & \\
\hline 6 & if $\mathbf{D}$ & honest & & & & \\
\hline 8 & if not vd & partially dishonest & & & + & - \\
\hline 14 & if not $\mathbf{V d}$ & partially dishonest & & - & - & + \\
\hline
\end{tabular}

$a$ : The number of times that the equilibria requirements violate $(-)$ or match $(+)$ the assumptions of the four effects described in the text. 1 = Pleiotropy between colour and defenses; 2 = Defenses affect the fitness of the tree after insect attack; $3=$ Vigour affects the cost of the signal; $4=$ Vigour affects the fitness of the tree after insect attack. 